\title{
Quantitative genetic analysis suggests causal association between cuticular hydrocarbon composition and desiccation survival in Drosophila melanogaster
}

\author{
BR Foley ${ }^{1}$ and M Telonis-Scott ${ }^{2}$ \\ ${ }^{1}$ Department of Biological Sciences, University of Southern California, Los Angeles, CA, USA and ${ }^{2}$ Department of Genetics, \\ The Centre for Environmental Stress and Adaptation Research, University of Melbourne, Parkville, Australia
}

\begin{abstract}
Survival to low relative humidity is a complex adaptation, and many repeated instances of evolution to desiccation have been observed among Drosophila populations and species. One general mechanism for desiccation resistance is Cuticular Hydrocarbon ( $\mathrm{CHC}$ ) melting point. We performed the first Quantitative Trait Locus (QTL) map of population level genetic variation in desiccation resistance in D. melanogaster. Using a panel of Recombinant Inbred Lines (RILs) derived from a single natural population, we mapped QTL in both sexes throughout the genome. We found that in both sexes, CHCs correlated strongly with desiccation resistance. At most desiccation resistance loci there was a significant association between $\mathrm{CHCs}$ and
\end{abstract}

desiccation resistance of the sort predicted from clinal patterns of $\mathrm{CHC}$ variation and biochemical properties of lipids. This association was much stronger in females than males, perhaps because of greater overall abundance of $\mathrm{CHCs}$ in females, or due to correlations between $\mathrm{CHCs}$ used for waterproofing and sexual signalling in males. $\mathrm{CHC}$ evolution may be a common mechanism for desiccation resistance in $D$. melanogaster. It will be interesting to compare patterns of $\mathrm{CHC}$ variation and desiccation resistance in species which adapt to desiccation, and rainforest restricted species which cannot.

Heredity (2011) 106, 68-77; doi:10.1038/hdy.2010.40; published online 14 April 2010

Keywords: D. melanogaster, QTL; pleiotropy; desiccation; CHCs

\section{Introduction}

The genetics of complex adaptation, particularly the degree to which populations follow either parallel or divergent evolutionary trajectories under common selective pressures is an area of active interest in evolutionary biology see (Gompel and Prud'homme, (2009); Stern and Orgogozo, 2009). Desiccation resistance in Drosophila is an interesting candidate complex trait in which to study repeated patterns of evolution among species and populations (Hoffmann and Harshman, 1999; Gibbs, 2002). Many Drosophila encounter periods of low humidity during their life-history and must balance water content against integumentary water loss owing to a high surface area/volume ratio. In doing so, numerous Drosophila species have successfully colonized arid habitats including deserts and high altitudes as well as tropical and temperate zones, providing an excellent model to study adaptation to desiccation at the intra- and inter-population level (Parsons, 1991; Gibbs, 2002; Parkash et al., 2005). By contrast several rainforest restricted species show little ability to

Correspondence: $\operatorname{Dr}$ BR Foley, Molecular and Computational Biology, University of Southern California, 1050 Childs Way, RRI 316, Los Angeles, CA 90007, USA.

E-mail:bradfole@usc.edu

Received 1 December 2009; revised 18 February 2010; accepted 11

March 2010; published online 14 April 2010 evolve resistance to desiccation (Hoffmann et al., 2003; Kellermann et al., 2009), although they show ample genetic variation for many other traits. Little is known about the mechanisms underlying natural genetic variation for desiccation survival in Drosophila at the population scale, and thus why some species adapt easily whereas others are limited by low adaptive variation for desiccation stress.

To date, most studies on the evolution of desiccation resistance have focused on populations derived from experimental evolution, with emphasis on the physiological, correlated and life-history responses associated with increased desiccation reviewed in (Hoffmann and Harshman 1999; Telonis-Scott et al., 2006). Comparative physiology among different $D$. melanogaster lines suggests that multiple evolutionary solutions can arise from a common selection pressure, although reducing water loss by water retention is a common mechanism underlying survival to desiccation (Gibbs et al., 1997; Hoffmann and Harshman, 1999; Gibbs, 2002; Telonis-Scott et al., 2006). Although artificial selection experiments are not without limitations, trait variation in natural populations may be inferred if enough alleles are sampled from the founding population. Signatures of natural selection for desiccation resistance are also evidenced in latitudinal clines, where survival can vary markedly among Drosophila according to local climatic conditions. Substantial variation in survival between populations 
suggests local adaptation and the presence of ample genetic variation (Coyne et al., 1983; Blows and Hoffmann, 1993; Kennington et al., 2001; Hoffmann et al., 2005). Opposing clines for desiccation and starvation resistance have been observed for seven Drosophilid species of the Indian subcontinent including D. melanogaster, where desiccation survival increased with latitude (Parkash et al., 1994; Karan and Parkash, 1998; Karan et al., 1998; Parkash and Munjal, 1999)

In D. melanogaster, geographic clines in Cuticular Hydrocarbon (CHC) profiles parallel climatic clines and correlate with patterns of desiccation resistance (Rouault et al., 2001, 2004). CHCs are the main constituent of the insect epicuticle, which functions primarily as a barrier against desiccation in nature (Hadley, 1981). Hydrocarbon chains in D. melanogaster range in length from 20-34 carbons, and there is evidence to suggest that expression of different chain lengths can affect survival to abiotic stresses such as desiccation and temperature (Toolson and Kupersimbron, 1989; Gibbs et al., 1991, 1997; Gibbs, 1998). Whether genetic variation in CHC expression at the population level in Drosophila correlates with variation in desiccation resistance remains to be seen, and it is unclear whether, despite the dimorphism in males and females, they use similar genetic mechanisms to adapt to common stresses.

Here, we use Quantitative Trait Locus (QTL) analyses in a set of recombinant inbred lines (RILs) to explore associations between survival to desiccation stress and CHCs at the population level in male and female D. melanogaster. QTL methodology permits statistical analyses of the associations between phenotype and genotype to characterize a minimum set of genomic regions that affect complex traits (Doerge, 2002). Quantitative trait loci for many fitness-related, stress resistance and sexually dimorphic traits have previously been mapped in this particular panel of RILs, which originate from two wild caught heterozygous $D$. melanogaster isofemales (Kopp et al., 2003; Wang et al., 2004; Mezey et al., 2005). High levels of CHC variation have also been identified in these lines, and a large number of CHC QTL identified (Foley et al., 2007). Here, we use these RILs to characterize QTL underlying natural genetic variation for survival to desiccation, and report the first QTL data for this trait in D. melanogaster. Furthermore, we explore the causal relationship of CHC expression on desiccation resistance using a Structural Equation Modelling (SEM) framework (Li et al., 2006; Jansen et al., 2009).

\section{Materials and methods}

\section{Recombinant inbred lines (RILs) and $\mathrm{CHC}$ analysis}

A set of 144 RILs was generated from the cross between a single pair of $F_{1}$ progeny derived from two wild isofemales trapped at the same location (Winters, California). One hundred and fifty-two markers were retained for the analysis. The cross schematics and line genotyping are described in detail elsewhere see (Kopp et al., 2003). CHCs were assayed as described in Foley et al., 2007. Rearing and assay conditions were similar12/12 light/dark, 25C - for both the CHC assays and desiccation (below). Representative CHC traces for each sex are shown in Supplemental Figure 1 (reproduced, with permission from Foley et al., 2007).
Desiccation survival assays

Density was standardized in the RILs for two generations before the desiccation assays by placing five pairs of flies on dextrose cornmeal medium for 3 days (line, $n=103$ ). For the assays, flies from each RIL were collected across $2-3$ vials within $24-48 \mathrm{~h}$ of eclosion, matured for 2 days in a mixed sex cohort, then separated by sex and using $\mathrm{CO}_{2}$ anaesthesia and held at a density of five flies per vial for another day. Survival to desiccation was assessed by desiccating three replicates of five females and five males (sexes tested separately). The flies were placed in empty vials sealed with gauze and were affixed with Parafilm to another vial containing approximately $10 \mathrm{~g}$ of silica desiccant. Vials were scored at hourly intervals until all flies in a group had died $\left(\mathrm{LT}_{100}\right)$, and the time for half the flies to die $\left(\mathrm{LT}_{50}\right)$ was determined by linear interpolation. QTL mapping was performed for both sexes and time-points.

\section{Analysis of mean survival to desiccation}

The data for each trait was log transformed before analysis to normalize them (tested with proc UNIVARIATE, SAS Institute, Cary NC, USA, v9.1). Two-way ANOVAs were performed with sex as the fixed effect, and the line and line-by-sex as random effects. Genetic correlations between the traits and sexes were estimated (Proc CORR) after averaging between replicates.

\section{Composite interval mapping (CIM)}

QTL mapping software is designed to analyse mapping populations with two alleles derived from isogenic parents. As outbred founders were used in this study, up to four parental autosomal haplotypes, and 3 of the X, may be segregating among RILs. However, by adapting standard mapping software to the RILs used in this study, we can test whether an allele from one chromosome codes for a trait value significantly different from the average trait because of the weighted average of the other chromosomes described in detail in (Wang et al., 2004). Separate analyses were performed for each of the linkage groups; three for the $X$ and third chromosomes (two of the parental third chromosomes appear identical see Kopp et al., 2003), and four for the second chromosome. Female and male survival at 50\% mortality $\left(\mathrm{LT}_{50}\right)$ and $100 \%\left(\mathrm{LT}_{100}\right)$ mortality were analysed using the CIM procedure in Windows Version 2.0 QTL Cartographer (Basten et al., 1994, 1999). The CIM Model 6 of QTL Cartographer was used, with number of background markers $=2$, window size of $30 \mathrm{cM}$ and Kosambi mapping function. Significance thresholds of $\alpha=0.05$ were determined by 1000 permutations for each trait and chromosome. QTL analyses on other traits considered for estimation of pleiotropy with desiccation were performed in a similar way using the same software using identical marker sets.

\section{Results}

\section{Analysis of mean survival to desiccation}

The average time ( \pm s.d.) of $50 \%$ mortality to desiccation was 12.1 (4.25) and $7.4 \mathrm{~h}$ (1.93) for females and males, respectively. The average time ( \pm s.d.) for $100 \%$ mortality was $15.4 \mathrm{~h}$ (4.90) for females and 9.6 h (2.60) for males. Considering the sexes separately, the proportion of 
variance in desiccation resistance due to line (similar to broad sense heritability) was very high. For females, line explained $90.6 \%$ of the variance in $\mathrm{LT}_{50}$, and $90.7 \%$ of the variance in $\mathrm{LT}_{100}$, and in males 89.7 and $85.5 \%$, respectively. Table 1 presents the ANOVA for survival to desiccation. For both survival traits, there was a highly significant line and sex term in the ANOVA $(P<0.0001)$, as well as a significant line by sex interaction $(P<0.0001)$. All traits were genetically correlated both among and between the sexes, (Pearson correlations significant at $P<0.0001$ ): $r=0.73$ for male and female $\mathrm{LT}_{50} ; r=0.68$ for male and female $\mathrm{LT}_{100} ; r=0.95$ female $\mathrm{LT}_{50}$ and $\mathrm{LT}_{100}$, and $r=0.89$ for male $\mathrm{LT}_{50}$ and $\mathrm{LT}_{100}$.

\section{QTL analysis}

Composite interval mapping was employed to estimate the position of QTL affecting survival to desiccation in both males and females. The log-likelihood ratios (LOD) were plotted against the cytological position in Centimorgans (cM) for the 10 linkage groups across the three major chromosomes (Supplemental Figure 2). The

Table 1 Analysis of variance for survival to desiccation

\begin{tabular}{llrcrc}
\hline Trait & Source & d.f. & Mean squares & F-value & Significance \\
\hline Female LT 50 & Line & 103 & 42.417 & 9.63 & $P<0.0001$ \\
& Error & & 4.403 & & \\
Female LT 100 & Line & 103 & 57.222 & 9.74 & $P<0.0001$ \\
& Error & & 5.873 & & \\
Male LT $_{50}$ & Line & 102 & 8.965 & 8.67 & $P<0.0001$ \\
& Error & & 1.035 & & \\
Male LT $_{100}$ & Line & 102 & 14.544 & 5.88 & $P<0.0001$ \\
& Error & & 2.474 & & \\
$\mathrm{LT}_{50}$ & Line & 103 & 0.071 & 14.43 & $P<0.0001$ \\
& Sex & 1 & 6.898 & 1395.77 & $P<0.0001$ \\
& Line $\times$ sex & 102 & 0.013 & 2.65 & $P<0.0001$ \\
& Error & 397 & 0.005 & & \\
$\mathrm{LT}_{100}$ & Line & 103 & 0.064 & 13.12 & $P<0.0001$ \\
& Sex & 1 & 6.708 & 1376.60 & $P<0.0001$ \\
& Line $\times$ sex & 102 & 0.013 & 2.70 & $P<0.0001$ \\
& Error & 397 & 0.005 & & \\
\hline
\end{tabular}

separate CIM analysis of each LG for 50\% ( $\left.\mathrm{LT}_{50}\right)$ and $100 \%\left(\mathrm{LT}_{100}\right)$ female and male mortality are presented in (Supplemental Figure 2). As up to four alleles may be segregating among the RILs, QTL Cartographer was used to test if an allele significantly affected survival to desiccation compared with the weighted average of several alleles, and the positive or negative effects of a QTL on survival were estimated and expressed as the additive effect (Table 2).

Approximately 95\% confidence intervals were constructed for all significant peaks using the two-LOD support rule (Lynch and Walsh, 1998). As a conservative estimate, we assumed that significant peaks across adjacent marker intervals with overlapping confidence intervals were potentially a single QTL and where two QTL had non-overlapping confidence intervals, a minimum of two QTL were assumed to be in the region. Significant LOD maxima with wide confidence intervals spanning the entire linkage group were considered uninformative, and excluded from this analysis. The same QTL may be identified by the separate analysis of each chromosome, therefore each significant peak may not necessarily reflect a unique QTL (Mezey et al., 2005). Following the selection criteria outlined in Mezey et al. (2005), a single putative QTL was declared for a trait if significant peaks were observed for overlapping marker regions in different linkage groups. A total of 40 analyses were performed for 10 linkage groups and four traits. To express all QTL and confidence intervals for desiccation survival on the cytogenic scale, they were plotted according to the proportion of their distance between flanking markers (following Foley et al., 2007).

An approximate minimum set of 15 putative QTL for survival to desiccation was identified by these criteria (Table 2). Nine QTL co-localized between the sexes with concordant effects for all traits, suggesting substantial non sex-specific genetic co-regulation for survival to desiccation with only minor differences between 50 and $100 \%$ mortality. Three sex-specific QTL were observed on the $X$ chromosome. The largest effects on desiccation resistance mapped to the 2 nd chromosome-according to our criteria there were at least $8 \mathrm{QTL}$, of 15 ,

Table 2 Summary of putative QTL and effects on survival to desiccation in males and females

\begin{tabular}{|c|c|c|c|c|c|}
\hline Putative QTL & Linkage group & $L O D$ max $(C I)$ female $L T_{50}$ & $\begin{array}{l}L O D \max (C I) \\
\text { female } L T_{100}\end{array}$ & $\begin{array}{l}L O D \max (C I) \\
\quad \text { male } L T_{50}\end{array}$ & $\begin{array}{l}L O D \max (C I) \\
\text { male } L T_{100}\end{array}$ \\
\hline QTL1 & $X-1$ & - & - & - & $\uparrow$ 12B (6E-17E) \\
\hline QTL2 & $x-2$ & $\downarrow 7 F(6 B-8 C)$ & - & - & - \\
\hline QTL3 & $\mathrm{X}-2$ & $\downarrow 8 \mathrm{~F}(8 \mathrm{D}-9 \mathrm{~A})$ & - & - & - \\
\hline QTTL4 & $2-2$ & $\downarrow 23 \mathrm{C}(22 \mathrm{E}-35 \mathrm{~F})$ & $\downarrow 23 \mathrm{C}(22 \mathrm{E}-35 \mathrm{~F})$ & - & - \\
\hline QTL5 & $2-1$ & $\uparrow 25 \mathrm{~B}(21 \mathrm{~A}-30 \mathrm{~A})$ & $\uparrow 25 B(21 C-32 C)$ & $\uparrow 25 \mathrm{~B}(22 \mathrm{C}-33 \mathrm{~F})$ & $\uparrow 27 \mathrm{D}(21 \mathrm{C}-33 \mathrm{~F})$ \\
\hline QTL6 & $2-3$ & - & - & $\uparrow 28 \mathrm{C}(25 \mathrm{~B}-31 \mathrm{E})$ & $\uparrow 28 \mathrm{C}(25 \mathrm{~B}-31 \mathrm{E})$ \\
\hline QTL6 & $2-2$ & $\downarrow 28 \mathrm{D}(26 \mathrm{D}-34 \mathrm{E})$ & $\downarrow 28 \mathrm{D}(26 \mathrm{D}-34 \mathrm{E})$ & $28 \mathrm{D}(23 \mathrm{C}-36 \mathrm{~B})$ & $\downarrow 28 \mathrm{D}(23 \mathrm{C}-36 \mathrm{~B})$ \\
\hline QTL7 & $2-2$ & $\downarrow 31 \mathrm{C}(28 \mathrm{~F}-33 \mathrm{~B})$ & $\downarrow 31 C(27 C-33 B)$ & $31 \mathrm{C}(25 \mathrm{~B}-34 \mathrm{~B})$ & $31 \mathrm{C}(25 \mathrm{~B}-34 \mathrm{~B})$ \\
\hline QTLL8 & $2-2$ & $\downarrow 37 \mathrm{C}(22 \mathrm{~A}-47 \mathrm{C})$ & $\downarrow 37 \mathrm{C}(22 \mathrm{~A}-45 \mathrm{C})$ & $\downarrow 37 \mathrm{C}(22 \mathrm{~A}-55 \mathrm{~A})$ & $37 \mathrm{C}(23 \mathrm{C}-49 \mathrm{~F})$ \\
\hline QTL9 & $2-1$ & $\uparrow 45 \mathrm{C}(36 \mathrm{~A}-53 \mathrm{E})$ & $\uparrow 44 \mathrm{~A}(22 \mathrm{C}-53 \mathrm{E})$ & $\uparrow 40 \mathrm{~A}(21 \mathrm{C}-53 \mathrm{E})$ & $\uparrow 40 \mathrm{~A}(21 \mathrm{C}-48 \mathrm{D})$ \\
\hline QTL10 & $2-3$ & $\downarrow$ 47D-E (43E-47E) & $\downarrow$ 47D-E (45D-47E) & $\downarrow$ 47D-E (42A-47E) & $\downarrow$ 47D-E (22B-47E) \\
\hline QTL11 & $2-1$ & $\uparrow 51 \mathrm{C}(39 \mathrm{E}-52 \mathrm{~F})$ & $\uparrow 51 \mathrm{C}(50 \mathrm{~A}-52 \mathrm{~F})$ & $\uparrow 51 \mathrm{C}(21 \mathrm{C}-52 \mathrm{~F})$ & - \\
\hline QTTL12 & $3-2 / 3$ & - & - & - & $\uparrow 71 \mathrm{E}-76 \mathrm{C}$ (66D-77D) \\
\hline QTL13 & $3-2 / 3$ & $\uparrow$ 84D-F (77C-85D) & $\uparrow$ 84D-F (77C-84F) & $\uparrow$ 84D-F (83C-85D) & $\uparrow$ 84D-F (83C-85F) \\
\hline QTL13 & $3-1$ & - & - & - & $\downarrow 84 \mathrm{DE}(69 \mathrm{~A}-84 \mathrm{DE})$ \\
\hline QTTL14 & $3-1$ & $\downarrow$ 87A (87A-89AB) & $\downarrow$ 87A-F (87A-97B) & $\downarrow$ 87A (87A-94D) & $\downarrow$ 87F-F (87A-94D) \\
\hline QTL15 & $3-1$ & - & - & - & $\downarrow$ 89AB (86D-94D) \\
\hline
\end{tabular}

The LOD maxima for each interval and overlapping 2-LOD support intervals (in brackets) are given for all traits. Arrows indicate the QTL effect on survival. Desiccation QTL with overlapping CHC QTL (Foley et al, 2007) are indicated in bold. 
segregating on chromosome 2, 6 of which co-localized between males and females (Table 2, Supplemental Figure 2). The largest effect QTL were identified on the distal end of $2 \mathrm{~L}$ for linkage groups $2-1,2-2$ and 2-3 between cytological positions 22-33, but for both sexes, there were QTL on all the major chromosomes.

There was qualitative QTL agreement between several desiccation loci and CHC expression (Foley et al., 2007) across chromosome 2L, and many of these QTL colocalized in both sexes (Table 2, Supplemental Figure 2 ). On chromosome 3 and the $X$ chromosome, male and female CHC expression did not colocalize well with each other, or with desiccation QTL. Although there is a large desiccation QTL on 3L on linkage group 3-2\&3 for both males and females, we observed no significant CHC QTL on male 3-2\&3. Similarly, while on the 3R chromosomal arm of linkage group 3-1 there are QTL for desiccation resistance in males and females, there are no female $\mathrm{CHC}$ QTL. Because confidence intervals for $\mathrm{CHC}$ traits and desiccation were so broad, however, it is impossible to assess pleiotropy based on colocalization.

\section{$\mathrm{CHC}$ and desiccation pleiotropy}

Principal Components (PC) analysis is commonly used as a factor reduction method to study large numbers of correlated traits (Stevens, 1996). Because PCs are constrained to be uncorrelated, they are also ideal for use in multiple regression analysis, to avoid overfitting and because multicollinearity can make it difficult to assign significance to particular factors. We conducted PC analyses on the covariance matrix of male and female CHC logcontrasts (Supplemental Table 1). Logcontrasts are a standard transformation used in the previous analysis of CHCs in these lines (Foley et al., 2007). We plotted the eigenvalues on a scree plot to determine, which PCs explained the majority of the non-error variance in CHC expression (Stevens, 1996). In males, the top three PCs were retained, and in females the top five. Multiple regression of the major PCs on desiccation showed that $\mathrm{CHCs}$ and desiccation resistance were highly correlated in both sexes, and that CHCs explained from $31-45 \%$ of variation in desiccation resistance in these lines. For female $\mathrm{LT}_{50}$ both PC2 and PC3 were highly significant $\left(\mathrm{PC} 2_{1,101} t=4.94, P<0.001 ; \mathrm{PC}_{1,101}\right.$, $t=-5.696, P<0.001)$ with a model adjusted $r^{2}$ of 0.341 . For $\mathrm{LT}_{100}, \mathrm{PC} 2$ and PC3 were also both significant (PC2 1 , $\left.101 t=-3.97, P<0.001 ; \mathrm{PC}_{1}, 101 t=-5.96, P<0.001\right)$ with a total model adjusted $r^{2}$ of 0.324 . In males PC1, PC2 and PC3 were all significantly associated with both $\mathrm{LT}_{50} \quad\left(\mathrm{PC}_{1,103}=-2.38, P=0.019 ; \quad \mathrm{PC} 2_{1,103}, \quad t=-3.57\right.$, $\left.P=0.001 ; \mathrm{PC}_{1,101}, t=-3.24, P=0.002\right)$ with an adjusted $r^{2}$ of 0.203 , and $\mathrm{LT}_{100}\left(\mathrm{PC}_{1,103}=-4.10, P<0.001 ; \mathrm{PC}_{1,103}\right.$, $\left.t=-4.44, P<0.001 ; \mathrm{PC}_{1,101}, t=-3.80, P<0.001\right)$ and an adjusted $r^{2}$ of 0.32 . Lower PCs were not significantly associated with desiccation resistance in either sex (data not shown)

\section{Correlations between traits}

It can be difficult to interpret loadings of PCs when looking for causal relationships between traits ( $\mathrm{Li}$ et al., 2006). We do, however, have a priori knowledge of the chemical classes of CHCs, and some idea of the expected mechanism by which they affect desiccation resistance in insects c.f. (Rouault et al., 2004). Therefore, to determine
Table 3 Correlations ( $r$ ) between desiccation and $\mathrm{CHC}$ traits measured in the RILs and their significance in female (a) and male (b) D. melanogaster

\begin{tabular}{|c|c|c|c|c|c|c|c|}
\hline & lt50 & $l t 100$ & Long & Linear & 7,11 & $7 C$ & Melt \\
\hline \multicolumn{8}{|l|}{ (a) } \\
\hline 1t50 & 1.000 & 0.960 & 0.525 & 0.269 & -0.273 & 0.093 & 0.377 \\
\hline Lt100 & $<0.001$ & 1.000 & 0.540 & 0.249 & -0.237 & 0.076 & 0.379 \\
\hline Long & $<0.001$ & $<0.001$ & 1.000 & 0.033 & 0.080 & -0.115 & 0.867 \\
\hline Linear & 0.007 & 0.012 & 0.723 & 1.000 & -0.747 & -0.135 & 0.304 \\
\hline 7,11 & 0.006 & 0.017 & 0.382 & $<0.001$ & 1.000 & -0.494 & -0.372 \\
\hline 7C & 0.356 & 0.448 & 0.208 & 0.141 & $<0.001$ & 1.000 & -0.035 \\
\hline \multirow[t]{2}{*}{ Melt } & $<0.001$ & $<0.001$ & $<0.001$ & 0.002 & $<0.001$ & 0.731 & 1.00 \\
\hline & lt50 & lt100 & Long & Linear & $7 C$ & Methyl & Melt \\
\hline \multicolumn{8}{|l|}{ (b) } \\
\hline Lt50 & 1.000 & 0.854 & 0.193 & 0.182 & 0.017 & 0.005 & 0.314 \\
\hline Lt100 & $<0.001$ & 1.000 & 0.293 & 0.184 & -0.006 & 0.120 & 0.444 \\
\hline Long & 0.051 & 0.003 & 1.000 & -0.241 & -0.258 & 0.634 & 0.846 \\
\hline Linear & 0.067 & 0.063 & 0.007 & 1.000 & -0.456 & -0.312 & -0.016 \\
\hline 7C & 0.869 & 0.954 & 0.004 & $<0.001$ & 1.000 & -0.347 & -0.094 \\
\hline Methyl & 0.963 & 0.227 & $<0.001$ & $<0.001$ & 0.000 & 1.000 & 0.680 \\
\hline Melt & 0.001 & 0.000 & 0.000 & 0.877 & 0.345 & 0.000 & 1.000 \\
\hline
\end{tabular}

Correlations are shown above the diagonal, and significant correlations are in bold. $P$-values are indicated below the diagonal.

whether particular chemical classes of CHCs contribute to increased desiccation resistance, subsets of the CHCs of the most abundant categories were selected and their relationship with desiccation estimated. Because male and female $\mathrm{CHC}$ profiles qualitatively differ in $D$. melanogaster, somewhat different chemical classes were considered in the two sexes. In males, linear alkanes (linear), 7:Cn alkenes (7C), and $2 \mathrm{MeCn}$ methylated alkanes (methyl) were evaluated, and in females linear alkanes (linear), 7:Cn alkenes (7C) and 7,11:Cn alkadienes $(7,11)$. Besides the chemical group, the relative abundance of long and short chain CHCs was calculated for each sex. For females, compounds with 27 carbons or more represented approximately half the $\mathrm{CHC}$ blend, and were considered long chain CHCs (long). For males, the compounds with 25 or more carbons represented approximately half the total amount, and were considered long chain CHCs for males (long). This is consistent with long/short ratios considered in other studies in this species (Rouault et al., 2004). Other metrics of chain length, such as mean $\mathrm{CHC}$ carbon number, gave very similar results (data not shown). We opted for this binning-metric as it is a proportion calculated in an identical manner to the other chemical-class metrics, allowing us to analyse all CHC traits similarly. The proportion of the total CHC abundance for these separate categories was calculated, and arcsine transformed to approximate a normal distribution.

Linear regression on the arcsine transformed traits shows that the proportion of long-chain CHCs expressed is a good predictor of desiccation resistance in the RILs, whereas the chemical class of the compound is evidently less important (Table $3 a$ and $b$ ). The proportion of longchain hydrocarbons positively correlates with desiccation resistance in both sexes. Correlations ranged from $19 \%$ for male desiccation $l_{50}$ (marginally non-significant) to $54 \%$ for female $\mathrm{lt}_{100}$ (highly significant: $P<0.0001$ ). In females, the proportion of both alkanes and 7,11:Cn alkadienes were also significantly associated with desiccation 
resistance. Multiple regression and model selection using AIC suggests that while the proportion of long-chain hydrocarbons is the largest contributor to desiccation resistance in both sexes, other aspects of $\mathrm{CHC}$ expression profile were important contributors to overall ability to resist desiccation. For females, the model including the proportion of long-chain $\mathrm{CHCs}$ and 7,11:Cn alkadienes best explained the data for both $\operatorname{LT}_{50}\left(\mathrm{~F}_{2,97}=27.93\right.$, $\left.r^{2}(\mathrm{adj})=0.352, \quad P \ll 0.0001\right)$, and $\mathrm{LT}_{100} \quad\left(\mathrm{~F}_{2,97}=27.91\right.$, $r^{2}$ (adj) $\left.=0.352, P \ll 0.0001\right)$. For males, the model including the proportion of long-chain CHCs, linear alkanes and $7: \mathrm{Cn}$ alkenes best explained the data for both $\mathrm{LT}_{50}$ $\left(\mathrm{F}_{3,98}=5.65, r^{2}(\mathrm{adj})=0.121, P=0.001\right)$ and $\mathrm{LT}_{100}\left(\mathrm{~F}_{3}\right.$, $\left.98=9.32, \quad r^{2}(\mathrm{adj})=0.198, \quad P<0.0001\right)$. In females, the correlation of long-chain hydrocarbons and desiccation resistance was similar to the model adjusted $r$ of PCs on desiccation traits $\left(0.584\right.$ and 0.569 for $l_{50}$ and $l_{100}$, respectively), whereas in males the correlation was less strong than found in the PC regression. There were also significant correlations between different chemical $\mathrm{CHC}$ classes in females, and especially in males, as well as between male and female CHC traits (Supplemental Table 2). In particular, the correlation between male and female proportion of long-chain CHCs was relatively high, with an $r$ of 0.292 .

For females, the effect of chain length on desiccation resistance was positive, whereas that for 7,11:Cn alkadienes was negative. For males, the effect of all variables were positive. For a given chain length, the melting point of CHCs decreases with the number of double bonds or methyl groups (Supplemental Figure 1, reproduced from Foley et al., 2007). The direction of effects in the best-fit models were consistent with an interpretation that overall CHC melting point was important in determining desiccation resistance. Accordingly, we constructed a metric of melting point by taking the average retention time of CHCs (which is proportional to melting point) weighted by compound abundance, for each sex and genotype. Average melting point was a significant predictor of desiccation resistance for female $\mathrm{LT}_{50}$ $\left(\mathrm{F}_{1,98}=16.21, \quad r^{2}(\mathrm{adj})=0.133, \quad P=0.0001\right), \quad$ and $\mathrm{LT}_{100}$ $\left(\mathrm{F}_{1,98}=16.44, r^{2}(\mathrm{adj})=0.135, P=0.0001\right)$; as well as male $\mathrm{LT}_{50}\left(\mathrm{~F}_{1,100}=10.92, r^{2}(\mathrm{adj})=0.089, P=0.001\right)$, and $\mathrm{LT}_{100}$ $\left(\mathrm{F}_{1,100}=24.59, r^{2}(\mathrm{adj})=0.189, P \ll 0.0001\right)$. These $r^{2}$ values are somewhat less than the best-fit PC models. Model selection using AIC, including the other CHC traits, agrees that while the melting point metric explains the majority of the contribution of CHCs to desiccation resistance, there are other important interactions between $\mathrm{CHC}$ expression and desiccation resistance not captured by this metric. For females, the best fit models did not include melting point at all, and instead the model described above was favoured by AIC. For males, AIC favoured models, which included melting point and the proportion of methylated alkanes for both $\mathrm{LT}_{50}$ $\left(\mathrm{F}_{1,100}=10.81, \quad r^{2}\right.$ (adj) $\left.=0.17, \quad P<0.0001\right), \quad$ and $\mathrm{LT}_{100}$ $\left(F_{1,100}=18.12, r^{2}(\mathrm{adj})=0.25, P \ll 0.0001\right)$. In both cases, the proportion of methylated alkanes contributed significantly and negatively to desiccation resistance-this is in contrast with the univariate correlation (Table $3 b$ ).

\section{Mapping-based evidence of pleiotropy}

To verify the pleiotropy of shared QTL, we modelled the effect of $\mathrm{CHCs}$ on desiccation in a linear regression framework, and conducted CIM mapping of the residuals (Schadt et al., 2005). In complex networks of associated traits, within a Structural Equation Modelling (SEM) framework, mapping of residuals in this way may be used to determine the effect of one trait on the expression of another, and to infer the direction of causality throughout regulatory networks (Jansen et al., 2009, Li et al., 2006). As our network consists of only two traits, and we have functional knowledge of the relationship between CHC expression and desiccation resistance, we did not explore the inverse function-the effect of desiccation resistance on CHC expression. We separately examined both the relationship between CHC PCs, and the melting point CHCs on desiccation. For both sexes we modelled only the PCs with a significant effect on desiccation resistance. For females, we used the residuals of the model containing PCs 2 and 3; for males the model containing PCs 1, 2 and 3. We examined the effects of $\mathrm{CHC}$ melting point as opposed to other classes of $\mathrm{CHC}$ traits (such as the proportion of linear alkanes), because this was the best univariate contributor to desiccation resistance, and we have a priori theoretical reasons to expect $\mathrm{CHC}$ melting point to mechanistically affect desiccation resistance.

A 2 LOD drop between an original trait and its residual at a QTL peak is suggested as a general significance threshold for determining whether there is a significant causal relationship between a dependent trait and its predictor ( $\mathrm{Li}$ et al., 2006). As our data set differed in many ways from the simulated data sets used to establish the significance of a 2 LOD drop, we empirically tested for the significance of the LOD dropoff between the original traits and their residuals by permutation. We permuted $\mathrm{CHC}$ traits relative to desiccation among the lines 1000 times, and modelled the effects of the permuted CHC traits on desiccation as with the original traits, and extracted the residuals. We mapped the residuals in QTL cartographer, using the same settings as above. Within the marker intervals flanking each of the original QTL LOD maxima, we took the highest LOD score for each of the permuted residuals and ranked them. An LOD drop between the original trait and residuals more extreme than 95\% of the residuals from the permuted data set is taken to indicate the drop is significant at $P=0.05$. An LOD drop more extreme than among all of the residuals from the permuted data set is taken to indicate the drop is significant at $P<0.001$.

We found pervasive effects of $\mathrm{CHC}$ expression on desiccation resistance throughout the genome in both sexes (Table 4, Figure 1). The majority of desiccation QTL, were in fact significantly reduced in effect when the effects of CHCs were removed (Table 4, Supplemental Figure 3). Across all QTL, we found that the two LOD dropoff criterion was very conservative relative to permutation. For females, CHCs were found to contribute to all desiccation QTL and the melting point of CHCs correlated positively with desiccation resistance at all QTL, apart from the QTL on 3-1. In males, the association between $\mathrm{CHCs}$ and desiccation somewhat less straightforward. Regression of CHC PCs on desiccation resistance indicated that $\mathrm{CHCs}$ contribute significantly to all identified QTL, similar to the case in females. At some QTL however, the association between desiccation and $\mathrm{CHC}$ melting point was in the opposite 
Table 4 The LOD maxima for the original desiccation QTL, as well as the LOD maxima in the same marker interval of their residuals, for both regression of CHC PCs and long-chain CHCs in D. melanogaster females (a) and males (b)

\begin{tabular}{|c|c|c|c|c|c|c|c|}
\hline & & $\mathrm{flt}_{50}$ & PC dropoff & Melt dropoff & $\mathrm{flt}_{100}$ & PC dropoff & Melt dropoff \\
\hline \multicolumn{8}{|l|}{ (a) } \\
\hline QTL1 & $X-1$ & - & - & - & - & - & - \\
\hline QTL2 & $X-2$ & 2.02 & $1.08(1000=1.14)$ & $1.48(P=0.008)$ & - & - & - \\
\hline QTL3 & $X-2$ & 2.08 & $1.73(P=0.069)$ & $1.92(P=0.092)$ & - & - & - \\
\hline QTL5 & $2-1$ & 6.27 & $1.69(1000=3.93)$ & $4.83(P=0.002)$ & 6.96 & $1.95(1000=4.69)$ & $5.19(P=0.001)$ \\
\hline QTL9 & $2-1$ & 6.46 & $4.76(P=0.001)$ & $4.63(1000=5.04)$ & 6.74 & $4.62(P=0.002)$ & $4.75(P=0.002)$ \\
\hline QTL11 & $2-1$ & 6.66 & $3.54(1000=4.67)$ & $3.52(1000=4.96)$ & 6.77 & $3.31(1000=4.59)$ & $1.90(1000=4.90)$ \\
\hline QTL4 & $2-2$ & 2.05 & $2.52(P=0.605)$ & $1.98(P=0.161)$ & 3.92 & $2.35(P=0.015)$ & $2.03(P=0.003)$ \\
\hline QTL6 & $2-2$ & 4.21 & $3.81(1000=4.02)$ & $3.81(1000=4.42)$ & 9.00 & $5.29(P=0.002)$ & $4.98(1000=5.54)$ \\
\hline QTL7 & $2-2$ & 7.18 & $2.82(1000=5.25)$ & $3.50(1000=5.76)$ & 8.38 & $3.67(1000=5.81)$ & $4.32(1000=6.74)$ \\
\hline QTL88 & $2-2$ & 2.36 & $1.28(1000=1.52)$ & $0.91(1000=2.29)$ & 2.56 & $1.34(P=0.002)$ & $1.04(1000=1.77)$ \\
\hline QTL6 & $2-3$ & - & - & - & - & - & - \\
\hline QTL10 & $2-3$ & 2.79 & $2.07(P=0.005)$ & $2.10(1000=2.11)$ & 2.87 & $2.11(P=0.004)$ & $2.19(P=0.003)$ \\
\hline QTL13 & $3-1$ & - & - & - & - & - & - \\
\hline QTL14 & $3-1$ & 2.46 & $0.39(1000=0.72)$ & $2.96(P=0.993)$ & 2.16 & $0.28(1000=0.51)$ & $2.74(0=2.94)$ \\
\hline QTL15 & $3-1$ & - & - & - & - & - & - \\
\hline QTL12 & $3-2 / 3$ & - & - & - & - & - & - \\
\hline \multirow[t]{2}{*}{ QTTL13 } & $3-2 / 3$ & 3.23 & $0.18(1000=0.81)$ & $0.70(1000=1.45)$ & 3.82 & $0.27(1000=2.42)$ & $1.00(1000=2.56)$ \\
\hline & & mlt $_{50}$ & PC dropoff & Melt dropoff & $\mathrm{mlt}_{100}$ & PC dropoff & Melt dropoff \\
\hline \multicolumn{8}{|l|}{ (b) } \\
\hline QTL1 & $X-1$ & - & - & - & 2.15 & $1.59(P=0.003)$ & $1.06(1000=2.52)$ \\
\hline QTL2 & $X-2$ & - & - & - & - & - & - \\
\hline QTL3 & $X-2$ & - & - & - & - & - & - \\
\hline QTL5 & $2-1$ & 4.11 & $1.25(1000=2.22)$ & $3.75(P=0.100)$ & 4.11 & $1.26(1000=2.18)$ & $3.96(P=0.310)$ \\
\hline QTL9 & $2-1$ & 2.77 & $1.11(1000=1.18)$ & $2.42(P=0.573)$ & 2.08 & $0.66(1000=1.22)$ & $1.82(P=0.032)$ \\
\hline QTL11 & $2-1$ & 1.99 & $0.62(1000=0.90)$ & $1.64(P=0.272)$ & - & - & - \\
\hline Q̄TL4 & $2-2$ & - & - & - & - & - & - \\
\hline QTL6 & $2-2$ & 2.05 & $1.60(1000=1.64)$ & $1.70(P=0.009)$ & 3.49 & $1.72(1000=2.256)$ & $1.70(1000=2.84)$ \\
\hline QTL7 & $2-2$ & 2.94 & $1.49(1000=1.08)$ & $1.73(1000=1.92)$ & 3.71 & $1.64(1000=2.578)$ & $1.72(1000=2.71)$ \\
\hline QTL8 & $2-2$ & 1.93 & $0.82(1000=0.92)$ & $1.19(1000=1.25)$ & 2.59 & $1.08(1000=1.337)$ & $1.52(P=0.001)$ \\
\hline QTL6 & $2-3$ & 2.20 & $0.71(P=0.003)$ & $1.22(0=1.10)$ & 1.37 & $0.11(1000=0.823)$ & $1.23(0=1.07)$ \\
\hline QTL10 & $2-3$ & 1.84 & $1.54(P=0.587)$ & $0.57(1000=0.62)$ & 1.03 & $0.45(P=0.004)$ & $0.73(P=0.261)$ \\
\hline QTL13 & $3-1$ & - & - & - & 3.45 & $0.26(P=0.016)$ & $2.72(P=0.024)$ \\
\hline QTL14 & $3-1$ & 1.78 & $0.04(1000=0.13)$ & $0.13(1000=1.04)$ & 3.67 & $0.24(P=0.005)$ & $1.14(P=0.001)$ \\
\hline QTL15 & $3-1$ & - & - & - & 2.73 & $0.18(P=0.002)$ & $0.40(P=0.005)$ \\
\hline QTL12 & $3-2 / 3$ & - & - & - & 2.83 & $1.02(1000=1.309)$ & $2.39(P=0.048)$ \\
\hline QTL13 & $3-2 / 3$ & 3.37 & $1.16(1000=2.16)$ & $2.68(P=0.001)$ & 4.09 & $1.18(1000=2.342)$ & $2.80(P=0.001)$ \\
\hline
\end{tabular}

$P$-values determined by 1000 permutations of $\mathrm{CHC}$ traits on desiccation resistance, and QTL mapping of the residuals are shown in brackets in which the LOD maxima of residuals are more extreme than any of the permuted-trait residuals, the most extreme values of the 1000 are noted in brackets.

Significant values as determined by permutation are indicated in bold.

Significant values as determined by 2 LOD dropoff are indicated in italics.

direction to that we would expect-notably on chromosome 2, linkage group 3, where the LOD of the residuals are significantly higher than the original trait. This indicates that the effect of $\mathrm{CHCs}$ at these loci were in the opposite direction as the overall trend of the regression (Li et al., 2006).

The association between female desiccation resistance and $\mathrm{CHC}$ expression was higher for almost all metrics, and this was supported by the results of the QTL mapping. Females expressed significantly more CHCs overall, however- 1.27 times the total amount of CHCs as males $\left(\mathrm{F}_{1,246}, \mathrm{~F}=15.98, P>0.001\right)$.

\section{Candidate genes}

We considered genes from the literature known to affect hydrocarbon profile as candidate genes (Supplemental Table 3), as well as genes identified through FlyBase $<$ http://flybase.org/ > containing Gene Ontology (GO) terms with the words 'fatty acid' or 'lipid', and 'metabolism', 'transport', 'synthase' or 'synthesis', and their equivalents. We also searched for the genes annotated with 'elongase', 'desaturase' and 'adult fat body'. These terms will necessarily miss many genes which, for instance, underlie fatty-acid or other resource allocation between tissues, or the many other possible ways in which energy and resource use might affect $\mathrm{CHC}$ profile. Given how broad many of the QTL are, each QTL is likely to contain thousands of genes, and multiple candidate and non-candidate genes potentially contribute to each QTL. Several candidates did colocalize with QTL (Supplemental Table 3).

\section{Discussion}

We present the first systematic study of population level genetic variation for survival to desiccation in D. melanogaster. Variation in resistance to desiccation was abundant among the RILs and was associated with multiple loci throughout the genome. We found high 

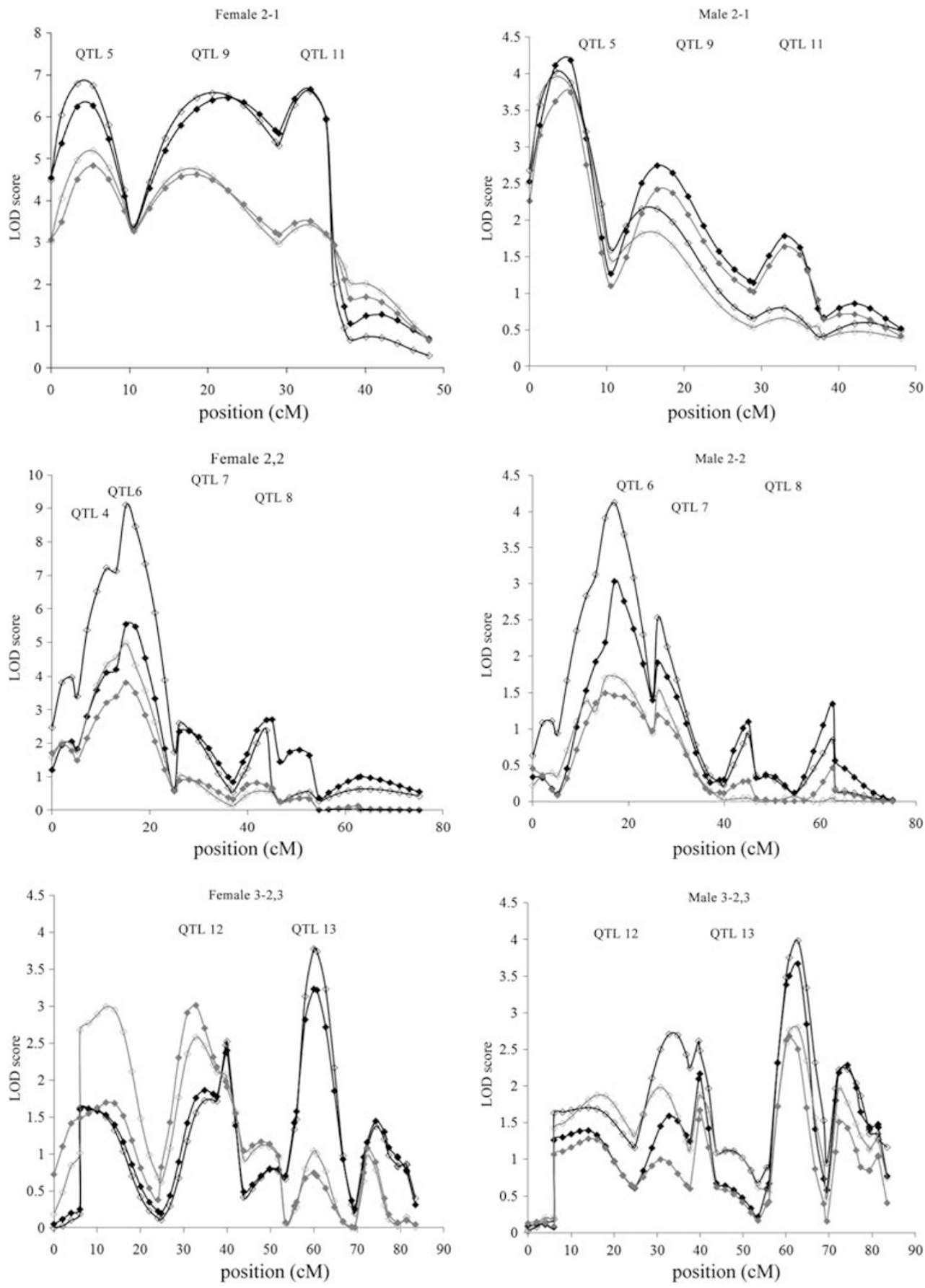

Figure 1 LOD plot of CIM map of desiccation traits (black lines) lt50 (solid symbols) and lt100 (open symbols); and their associated residuals (grey lines) of the regression of CHC melting point for RILs of D. melanogaster. Shown are the three linkage groups where $>2$ LOD drop-offs were found.

correlations between desiccation resistance and a previously hypothesized mechanistic trait (CHCs) in both sexes. This correlation was evident overall, and at specific loci throughout the genome.

CIM mapping identified at least 15 desiccation QTL, which most likely underestimates the number of genes underlying this trait discussed in (Mezey et al., 2005). Resistance to desiccation stress had a similar genetic basis in males and females, reflected in highly significant Pearson correlations of approximately $70 \%$ between the sexes. There was also evident co-localization of QTL between the sexes. The locus with the strongest effect was restricted to a region on the distal end of chromosomal arm 2L in both sexes, although with a much stronger effect in females, and large regions across the 3rd chromosome had effects on desiccation resistance with similar directionality of effects in males and females. The number of broad, but very significant regions of the genome associated with desiccation resistance is consistent with previous studies, which have shown a great deal of variation for resistance to low humidity in $D$. melanogaster selection experiments, with 
both X-linked and autosomal effects (Hoffmann and Parsons, 1989b; Telonis-Scott et al., 2006). Heritability estimates of around $60 \%$ for desiccation resistance (Hoffmann and Parsons, 1989b) are known, and at least in D. melanogaster, rapid responses to selection are usual (Hoffmann and Parsons, 1989aHoffmann and Parsons, 1993; Bubliy and Loeschcke, 2005; Telonis-Scott et al., 2006).

Differences between desiccation QTL at the two time points $\left(\mathrm{LT}_{50}\right.$ and $\left.\mathrm{LT}_{100}\right)$ were negligible, with only one QTL at 7F specific to female survival at 50\% mortality. In the other cases where QTL are listed for only one time point, the corresponding time-point showed a significant peak but was not included in the overall summary owing to low statistical support from wide confidence intervals (Table 2). Although the traits have a similar genetic basis in almost all cases, these loci do not have identical effects on both traits, and it may be that different alleles are associated with different stages during desiccation.

Water retention is a key adaptation to desiccation stress in Drosophila, where water is lost via excretion across the spiracles or cuticle; (Hoffmann and Harshman, 1999; Gibbs et al., 2003). In highly desiccation-resistant xeric species, water is retained primarily via lowered metabolic rate reducing respiration across the spiracles and changes in cyclic $\mathrm{CO}_{2}$ release (Gibbs et al., 2003). Changes in $\mathrm{CO}_{2}$ release via the spiracles was observed in selected lines of D. melanogaster (Williams et al., 1998), although the effect on desiccation was less clear. Initial associations between desiccation resistance and lowered metabolic rate in Drosophila selected lines tended to disappear when corrected for energy stores (Hoffmann and Parsons, 1989ab; Djawdan et al., 1998). Other mechanisms known to affect desiccation resistance include greater dehydration tolerance, increased bulk water, greater metabolic stores increased body size see (Telonis-Scott et al., 2006). In some cases several mechanisms have been found to contribute to desiccation resistance; that is, adipose variants when combined with wild-type alleles survived desiccation in part because of larger size, reduced transpiration and greater tolerance of low water content (Clark and Doane, 1983).

In terms of cuticular transpiration, it is known that CHCs are important for maintaining the impermeability of the cuticle, and that the melting point of the hydrocarbons, which increases with carbon number, is a factor in reducing water exchange through the cuticle (Gibbs et al., 1997; Gibbs 1998). Desiccation susceptibility owing to rapid water loss across the cuticle was documented in a desiccation sensitive mutant as well as lines selected for postponed senescence (Kimura et al., 1985; Graves et al., 1992). Here, consistent with theoretical predictions (Toolson and Kupersimbron, 1989) and global clinal patterns of CHC expression variation as well as plastic responses to temperature (Rouault et al., 2004), there were strong correlations between desiccation resistance and $\mathrm{CHC}$ melting point. In both sexes, these correlations were strong, up to $48 \%$ in females and $40 \%$ in males for desiccation $\mathrm{lt}_{100}$. In neither sex did the proportion of other chemical classes of CHCs predict desiccation resistance as well.

We found strong effects of CHCs on desiccation resistance at individual QTL. There were significant drops in the likelihood of nearly all desiccation QTL when the overall effects of $\mathrm{CHC}$ expression were removed. This significantly reduced the genomic landscape specific to desiccation and reflects that genetic variation for CHCs may be causal to the desiccation survival phenotype, itself a composite trait. The effect was clearer in females, where the magnitude of the drop in LOD was much larger than in males. Male desiccation LOD maxima were not generally as high, however, and this might simply reflect higher error variance in male measurements, consistent with overall higher LOD maxima in female CHC QTL (Foley et al., 2007), or the greater abundance of female CHCs. Although the 2 LOD significance threshold indicated effects of CHCs only at female desiccation QTL, permutation tests indicate the 2 LOD threshold was far too conservative in our panel of RILs. Permutation indicated that both male and female desiccation survival were affected by CHCs at many loci throughout the genome. Given that we have found many regions throughout the genome with effects on $\mathrm{CHC}$ expression (Foley et al., 2007), and given the predicted association between CHCs and desiccation resistance, this perhaps should not be surprising. We evidently have stronger power to detect the effects of genetic loci on $\mathrm{CHC}$ expression when considering multivariate measures such as PCs or CHC chemical classes than when mapping $\mathrm{CHCs}$ as univariate traits. Significant associations between $\mathrm{CHC}$ expression and desiccation were found even in haplotypes such as 3-2,3, in which single CHCs mapped poorly (Foley et al., 2007).

The predicted association between $\mathrm{CHC}$ melting point and increased desiccation resistance was robustly evident at an individual QTL in our lines. This is true particularly in females where at nearly every QTL the melting point of CHCs correlate with desiccation resistance. In males, the association is somewhat less consistent across QTL. This sex-specificity of effects at the genomic level is consistent with the overall lesser correlation of male $\mathrm{CHC}$ melting point with desiccation resistance. This may be due to interactions between traits that we have not measured. Notably in our lines, methylalkane and long-chain CHC expression are strongly positively correlated and methyl-alkanes are present in high levels in males but not in females, suggesting they are involved with sexual signalling in D. melanogaster, as they are found to be in other Drosophila (Chenoweth and Blows, 2005). It might be that males who exert strong signalling effort are subject to tradeoffs for desiccation survival. The various correlations between methylalkane expression, $\mathrm{CHC}$ melting point and desiccation resistance might explain why at one locus on $2-3$, the association between male $\mathrm{CHC}$ melting point and desiccation resistance is significantly in the direction opposite to that expected. Alternatively, D. melanogaster has been found to facultatively modify $\mathrm{CHC}$ profile in response to stresses, like heat, which are likely to increase the risk of desiccation (Rouault et al., 2004), with males strongly upregulating their long chain CHCs. As we measured all our CHCs at $25^{\circ} \mathrm{C}$, and at relatively high relative humidity, we likely missed all sex-specific facultative responses that may have affected desiccation survival.

Given our number of potential candidate genes, it was notable which of several strong candidates did not contribute to any QTL. We might expect elongaseswhich increase $\mathrm{CHC}$ chain length-to be involved in desiccation resistance. One elongase, Elo68 $\alpha$, might 
potentially contribute to QTL12, but this desiccation QTL did not seem to be affected by CHC expression, and the association thus seems dubious. Another elongase, EloF, did not colocalize with a strong LOD maxima. Fst (Sinclair et al., 2007), which has been shown to respond to environmental stress including desiccation and cold, did not colocalize with any QTL. Although Desat2 (Greenberg et al., 2003)_potentially implicated in stress resistance-colocalizes with QTL14, there are effects in both sexes of $\mathrm{CHC}$ expression on desiccation resistance at this locus. As desat2 expression is female specific, it seems unlikely to be involved in the RILs. We did not measure gene expression in this study, however, thus cannot detect trans effects on gene expression for our candidates. Of the strongest desiccation QTL most influenced by CHC expression, those at the distal end of $2 \mathrm{~L}$ had several potentially interesting candidates, including FatP, a fatty acid transport molecule, and smoq (Ferveur and Jallon, 1996). Another noted CHC-expression gene, sept (Ferveur and Jallon, 1996), also falls within the $95 \%$ confidence interval of several QTL. However, the transition from QTL to QTN (quantitative trait nucleotide) requires fine-scale molecular dissection (that is, complementation tests, transgenic studies, gene expression, cloning) to elucidate the contribution of individual genes to desiccation survival.

\section{Conclusion}

Given the extent of variation in CHCs found in even this small genetic sample, and the robust association of this variation with desiccation resistance in our lines, it seems plausible that within D. melanogaster and other Drosophila, $\mathrm{CHC}$ evolution is likely to be a common adaptation to desiccation stress. It will be interesting to test this association in species, which do not evidence an ability to adapt to desiccation. Although rainforest species like D. bunnanda have a great deal of genetic variation in their CHCs at a population level (Van Homrigh et al. 2007), it will be necessary to test whether they have any variation in mean hydrocarbon chain length to begin to understand why they cannot avail of this mechanism of desiccation resistance

\section{Conflict of interest}

The authors declare no conflict of interest.

\section{Acknowledgements}

This work was conducted in the labs of AA Hoffmann at LaTrobe University, Melbourne AU; SV Nuzhdin, UC Davis, Davis USA; MW Blows, University of Queensland, Brisbane AU. This project was funded by a National Institute of Health Grant RO161733 to SVN, and Australian Research Council grants to AAH and MWB. We thank SVN, AAH and MWB for all their generous support.

\section{References}

Basten CJ, Weir BS, Zeng Z-B (1994). Proceedings of the 5th World Congress on Genetics Applied to Livestock Production: Computing Strategies and Software In: C Smith GSG B Benkel, J Chesnais, W Fairfull et al. EB Burnside (ed.) Guleph, Ontario, pp 65-66.
Basten CJ, Weir BS, Zeng Z-B (1999). QTL cartographer, version 1.13. Department of statistics, North Carolina State Universtiy: Raleigh, NC.

Blows MW, Hoffmann AA (1993). The genetics of central and marginal populations of Drosophila serrata. 1. Genetic variation for stress resistance and species borders. Evolution 47: 1255-1270.

Bubliy OA, Loeschcke V (2005). Correlated responses to selection for stress resistance and longevity in a laboratory population of Drosophila melanogaster. J Evol Biol 18: 789-803.

Chenoweth SF, Blows MW (2005). Contrasting mutual sexual selection on homologous signal traits in Drosophila serrata. Am Naturalist 165: 281.

Chippindale AK, Gibbs AG, Sheik M, Yee KJ, Djawdan M, Bradley TJ et al. (1998). Resource acquisition and the evolution of stress resistance in Drosophila melanogaster. Evolution 52: 1342-1352.

Clark AG, Doane WW (1983). Desiccation tolerance of the adipose60 mutant of Drosophila melanogaster. Hereditas 99: 165-175.

Coyne JA, Bundgaard J, Prout T (1983). Geographic variation of tolerance to environmental-stress in Drosophila pseudoobscura. Am Naturalist 122: 474-488.

Djawdan M, Chippindale AK, Rose MR, Bradley TJ (1998). Metabolic reserves and evolved stress resistance in Drosophila melanogaster. Physiol Zool 71: 584-594.

Doerge RW (2002). Mapping and analysis of quantitative trait loci in experimental populations. Nat Rev Genet 3: 43-52.

Foley B, Chenoweth SF, Nuzhdin SV, Blows MW (2007). Natural genetic variation in cuticular hydrocarbon expression in male and female Drosophila melanogaster. Genetics 175: 1465-1477.

Folk DG, Han C, Bradley TJ (2001). Water acquisition and partitioning in Drosophila melanogaster: effects of selection for desiccation-resistance. J Exp Biol 204: 3323-3331.

Ferveur JF, Jallon JM (1996). Genetic control of male cuticular hydrocarbons in Drosophila melanogaster. Genet Res 67: 211.

Gibbs AG. (1998). Water-proofing properties of cuticular lipids. Am Zool 38: 471-482.

Gibbs AG (2002). Water balance in desert Drosophila: lessons from non-charismatic microfauna. Comp Biochem Physiol A-Mol Integrative Physiol 133: 781-789.

Gibbs AG, Chippindale AK, Rose MR (1997). Physiological mechanisms of evolved desiccation resistance in Drosophila melanogaster. J Exp Biol 200: 1821-1832.

Gibbs AG, Fukuzato F, Matzkin LM (2003). Evolution of water conservation mechanisms in Drosophila. J Exp Biol 206: 11831192.

Gibbs A, Mousseau TA, Crowe JH (1991). Genetic and acclimatory variation in biophysical properties of insect cuticle lipids. Proc Natl Acad Sci USA 88: 7257-7260.

Gompel N, Prud'homme B (2009). The causes of repeated genetic evolution. Dev Biol 332: 36-47.

Graves JL, Toolson EC, Jeong C, Vu LN, Rose MR (1992). Desiccation, flight, glycogen and postponed senescence in Drosophila melanogaster. Physiol Zool 65: 268-286.

Greenberg AJ, Moran JR, Coyne JA, Wu CI (2003). Ecological adaptation during incipient speciation revealed by precise gene replacement. Science 302: 1754-1757.

Hadley NF (1981). Cuticular lipids of terrestrial plants and arthropods-a comparison of their structure, composition, and waterproofing function. Biol Rev Cambridge Philosophical Soc 56(Feb): 23-27.

Hoffmann AA, Hallas RJ, Dean JA, Schiffer M (2003). Low potential for climatic stress adaptation in a rainforest Drosophila species. Science 301: 100-102.

Hoffmann AA, Harshman LG (1999). Desiccation and starvation resistance in Drosophila: patterns of variation at the species, population and intrapopulation levels. Heredity 83: 637-643. 
Hoffmann AA, Parsons PA (1989a). An integrated approach to environmental-stress tolerance and life-history variationdesiccation tolerance in Drosophila. Biol J Linnean Soc 37: 117-136.

Hoffmann AA, Parsons PA (1989b). Selection for increased desiccation resistance in Drosophila melanogaster-additive genetic-control and correlated responses for other stresses. Genetics 122: 837-845.

Hoffmann AA, Parsons PA (1993). Direct and correlated responses to selection for desiccation resistance-a comparison of Drosophila melanogaster and D. simulans. J Evol Biol 6: 643-657.

Hoffmann AA, Shirriffs J, Scott M (2005). Relative importance of plastic vs genetic factors in adaptive differentiation: geographical variation for stress resistance in Drosophila melanogaster from eastern Australia. Functional Ecol 19: 222-227.

Jansen RC, Tesson BM, Fu JY, Yang YJ, McIntyre LM (2009). Defining gene and QTL networks. Curr Opin Plant Biol 12: 241-246.

Karan D, Dahiya N, Munjal AK, Gibert P, Moreteau B, Parkash $\mathrm{R}$ et al. (1998). Desiccation and starvation tolerance of adult Drosophila: Opposite latitudinal clines in natural populations of three different species. Evolution 52: 825-831.

Karan D, Parkash R (1998). Desiccation tolerance and starvation resistance exhibit opposite latitudinal clines in Indian geographical populations of Drosophila kikkawai. Ecol Entomol 23: 391-396.

Kellermann V, van Heerwaarden B, Sgro CM, Hoffmann AA (2009). Fundamental evolutionary limits in ecological traits drive drosophila species distributions. Science 325: 1244-1246.

Kennington WJ, Gilchrist AS, Goldstein DB, Partridge L (2001). The genetic bases of divergence in desiccation and starvation resistance among tropical and temperate populations of Drosophila melanogaster. Heredity 87: 363-372.

Kimura K, Shimozawa T, Tanimura T (1985). Water-loss through the integument in the desiccation- sensitive mutant, parched of Drosophila melanogaster. J Insect Physiol 31: 573-580.

Kopp A, Graze RM, Xu SZ, Carroll SB, Nuzhdin SV (2003). Quantitative trait loci responsible for variation in sexually dimorphic traits in Drosophila melanogaster. Genetics 163: 771-787.

Li RH, Tsaih SW, Shockley K, Stylianou IM, Wergedal J, Paigen B et al. (2006). Structural model analysis of multiple quantitative traits. Plos Genet 2: 1046-1057.

Lynch M, Walsh B (1998). Genetics Analysis of Quantitative Traits. Sinauer Associates Inc: Sunderland, MA, USA, pp 448-449.

Mezey JG, Houle D, Nuzhdin SV (2005). Naturally segregating quantitative trait loci affecting wing shape of Drosophila melanogaster. Genetics 169: 2101-2113.

Parkash R, Munjal AK (1999). Climatic selection of starvation and desiccation resistance in populations of some tropical drosophilids. J Zool System Evol Res 37: 195-202.
Parkash R, Sharma S, Sharma M (1994). Patterns of starvation and desiccation tolerance in Drosophila bipectinata and Drosophila malerkotliana. Biologisches Zentralblatt 113: 355-363.

Parkash R, Tyagi PK, Sharma I, Rajpurohit S (2005). Adaptations to environmental stress in altitudinal populations of two Drosophila species. Physiol Entomol 30: 353-361.

Parsons PA (1991). Biodiversity conservation under global climatic-change-the insect Drosophila as a biological indicator. Glob Ecol Biogeogr Lett 1: 77-83.

Rouault J, Capy P, Jallon JM (2001). Variations of male cuticular hydrocarbons with geoclimatic variables: an adaptative mechanism in Drosophila melanogaster? Genetica 110: 117-130.

Rouault JD, Marican C, Wicker-Thomas C, Jallon JM (2004). Relations between cuticular hydrocarbon (HC) polymorphism, resistance against desiccation and breeding temperature; a model for $\mathrm{HC}$ evolution in $D$. melanogaster and D. simulans. Genetica 120: 195-212.

Sinclair BJ, Gibbs AG, Roberts SP (2007). Gene transcription during exposure to, and recovery from, cold and desiccation stress in Drosophila melanogaster. Insect Molec Biol 16: 435-443.

Schadt EE, Lamb J, Yang X, Zhu J, Edwards S, GuhaThakurta D et al. (2005). An integrative genomics approach to infer causal associations between gene expression and disease. Nat Genet 37: 710-717.

Stern DL, Orgogozo V (2009). Is Genetic Evolution Predictable? Science 323: 746-751.

Stevens J (1996). Applied Multivariate Statistics for the Social Sciences. Lawrence Erlbaum Associates Inc. Mahwah, NJ, p 364.

Telonis-Scott M, Guthridge KM, Hoffmann AA (2006). A new set of laboratory-selected Drosophila melanogaster lines for the analysis of desiccation resistance: response to selection, physiology and correlated responses. J Exp Biol 209 1837-1847.

Toolson EC, Kupersimbron R (1989). Laboratory evolution of epicuticular hydrocarbon composition and cuticular permeability in Drosophila pseudoobscura-effects on sexual dimorphism and thermal-acclimation ability. Evolution 43 468-473.

Van Homrigh A, Higgie M, McGuigan K, Blows MW (2007). The depletion of genetic variance by sexual selection. Curr Biol 17: 528-532.

Wang MH, Lazebny O, Harshman LG, Nuzhdin SV (2004). Environment-dependent survival of Drosophila melanogaster: a quantitative genetic analysis. Aging Cell 3: 133-140.

Williams AE, Rose MR, Bradley TJ (1998). Using laboratory selection for desiccation resistance to examine the relationship between respiratory pattern and water loss in insects. J Exp Biol 201: 2945-2952.

Supplementary Information accompanies the paper on Heredity website (http://www.nature.com/hdy) 